\title{
Clinical manifestations and outcomes of COVID-19 in the paediatric population: a systematic review
}

\author{
Maha Jahangir *, Marrium Nawaz, Deedar Nanjiani, Mishal S Siddiqui
}

This article was published on 30 Sep 2020 at www.hkmj.org.

\section{A B S T R A C T}

Objective: Coronavirus disease 2019 (COVID-19), the respiratory illness caused by severe acute respiratory syndrome coronavirus 2 , has affected hundreds of thousands of people. We aim to report the distribution of cases, prevalence, and clinical, radiological, and laboratory signs and outcomes of COVID-19 in paediatric patients. Moreover, we intend to evaluate neonatal clinical outcomes. Hence, our age range of interest is 0 to 19 years.

Methods: A systematic literature review was conducted using the Medline database to identify papers published between 1 December 2019 and 9 April 2020 on COVID-19.

Results: The search identified 27 relevant scientific papers and letters. The review showed that the prevalence of COVID-19 in the paediatric population accounts for a small percentage of patients, whose clinical signs and symptoms are often milder than those of adults. Despite better prognosis and low mortality in children, the disease can progress to severe pneumonia in some cases, especially in the presence of co-morbidities. Children are likely to become a hidden source of infection because of their atypical presentation, and they may play a role in community transmission, leading to unfavourable outcomes. There is little evidence about intrauterine vertical transmission. As no vaccine or specific antiviral is currently available, management plans include supportive treatment.

Conclusion: As compared with that in adults, the presentation of COVID-19 in children is mild and has a better prognosis. Sufficient evidence regarding the probability of intrauterine vertical transmission could not be found, and further studies need to be conducted to establish this relationship.

\section{Hong Kong Med J 2021;27:35-45}

https://doi.org/10.12809/hkmj208646

M Jahangir *, MB, BS

M Nawaz, MB, BS

D Nanjiani, MB, BS

MS Siddiqui, MB, BS

Dow Medical College, Dow University of Health Sciences, Karachi, Pakistan

* Corresponding author: jahangirmaha@yahoo.com

\section{Introduction}

Severe acute respiratory system coronavirus-2 (SARS-CoV-2) outbreak emerged as a series of idiopathic cases of severe pneumonia in early December 2019, with the first report made to the regional office of the World Health Organization on 30 December 2019. ${ }^{1}$ The epidemiological curve of affected individuals rose steeply worldwide, thus leading to the outbreak being declared as a public health emergency of international concern on 30 January $2020^{1}$ and subsequently a pandemic on 11 March $2020 .^{2}$ As of 30 April 2020, 3090445 cases have been reported worldwide, ${ }^{3}$ with a mortality rate of $3.4 \%,{ }^{4}$ culminating in the deaths of 217769 individuals. $^{3}$

Like other members of the Coronaviridae family, SARS-CoV-2, which causes coronavirus disease 2019 (COVID-19), is a positive-sense singlestranded RNA virus with an icosahedral capsid that primarily affects the respiratory tract. ${ }^{5}$ Other members of the family have caused pandemics with similar clinical presentations, such as Middle
East respiratory syndrome-related coronavirus (MERS-CoV) and SARS-CoV. However, COVID-19 outnumbers both of the others in terms of cases and deaths, despite its lower mortality rate compared with SARS-CoV and MERS-CoV. ${ }^{6}$

The COVID-19 outbreak primarily affects adults, and the severity of disease increases in an age-dependent fashion. Additional risk factors include male sex and co-morbidities including diabetes, hypertension, and previous respiratory impairments. ${ }^{7}$ Children and adolescents comprise a relatively minor proportion of patients who have tested positive for the virus. Moreover, the paediatric body responds to the disease differently from the adult body. This leads to heterogeneous clinical presentation, disease severity, and mortality rates across the age spectrum.

A review of 72314 cases by the Chinese Centre for Disease Control and Prevention reported that the age-groups of 10 to 19 years and under 10 years contributed $1 \%$ each to the total disease burden. ${ }^{8}$ Children aged under 18 years have been reported to 


\section{兒科人群的2019冠狀病毒病臨床表現和療效： 系統綜述}

\author{
Maha Jahangir 、Marrium Nawaz 、 Deedar Nanjiani 、 \\ Mishal S Siddiqui
}

目的：2019冠狀病毒病（COVID-19）是由嚴重急性呼吸綜合症冠狀 病毒 2 型引起的呼吸系統疾病, 已影響達數十萬人。本文旨在報告兒 科患者的COVID-19病例分佈、現患率, 以及臨床、放射學和實驗室 體徵及療效, 也嘗試評估新生兒的臨床療效。因此, 我們的研究年齡 範圍介乎 0 至 19 歲。

方法：使用Medline數據庫進行系統性文獻綜述, 以識別2019年 12月 1日至2020年4月9日期間發表的COVID-19文獻。

結果：經搜索識別27篇相關科學論文和信函。本文説明COVID-19在 兒科人群中的現患率頗低, 其臨床症狀和體徵通常較成人輕微。儘管 兒童的預後較佳且死亡率較低, 但在某些情況, 尤其出現併發症的情 況下, COVID-19可惡化至嚴重肺炎。由於其非典型表現, 兒童可能 成為隱性感染源, 在社區傳播後可導致不良結果。有關宮內垂直傳播 的證據很少。由於目前尚無疫苗或特定抗病毒藥物, 因此支持治療屬 管理計劃的一環。

結論：與成人相比, 兒童於COVID-19的表現較輕微和預後較佳。找 不到有關宮內垂直傳播可能性的充分證據, 須作進一步研究。 comprise $1.2 \%$ of the total cases in Italy, ${ }^{9}$ whereas in the US and Madrid, Spain, the contribution of this age-group has been reported as $1.7 \%^{10}$ and $0.8 \%^{11}$, respectively. Statistics from Pakistan reveal that children aged 10 to 19 years comprise $7.25 \%$ of the total number of cases, and this age-group's mortality has been approximated as $0.52 \% .^{12}$ Although the outbreak appears to be stabilising or declining in certain areas of the world, many regions are still witnessing an upward trend or even a resurgence.

Increased incidence of asymptomatic carriage and milder symptoms may lead to a decreased need for testing in the paediatric population, especially in already burdened healthcare systems. Hence, this age-group may remain as a source of continued transmission, the magnitude of which remains unexplored. Thus, we aimed to review the characteristics and presentation of COVID-19 among children and describe any subtle characteristics that may strengthen the clinical suspicion of infection and prompt further testing. This may help to break the chain of transmission and effectively decrease the global burden of the pandemic.

\section{Methods}

Considering the date of the earliest confirmed report of COVID-19, we searched Medline for studies published from 1 December 2019 until 9 April 2020, with no language restriction and with a combination of the key search terms "coronavirus" OR "COVID-19" or "2019-nCoV" OR "SARS-CoV-2" AND "baby" OR "babies" OR "pediatric" OR "paediatric" OR "newborn" OR "neonate" OR "adolescent" OR "child" OR "children” OR "infant” OR "boy” OR "girl” OR "teenage". We used a comprehensive search strategy to identify the relevant studies. The screening process was conducted by two independent reviewers (MJ and $\mathrm{MN}$ ), and a third reviewer (DN) was consulted in the event of discrepancies. The articles were screened on the basis of title and abstract to assess their relevance to the aims of our study, followed by full-text screening. For each retrieved full-text article, we hand-searched and examined the citation chain for additional studies. The PubMed search identified 325 articles. After excluding 229 irrelevant articles, 96 full-text articles were reviewed, of which only 27 were included in this review. ${ }^{10,11,13-37}$

This systematic review was conducted in accordance with the PRISMA statement. ${ }^{38}$ An inclusive approach to eligibility assessment was taken. Studies were deemed eligible if they provided clinical data on COVID-19 in the paediatric population (ie, aged 0-19 years). Therefore, a few letters that provided original data were also included in the study. We excluded letters, case reports, editorials, opinion pieces, or studies from which no data from paediatric patients could be extracted, focused on other coronaviruses than COVID-19, epidemiological studies that provided no clinical findings, and those in other languages with no English translation. The Figure shows PRISMA chart outlining the search strategy.

\section{Results and Discussion}

The clinical manifestations and outcomes of COVID-19 in the paediatric population are summarised in Table 1. 10,11,14-16,18-30,33,39-41

\section{Age}

Korean data suggested that a 10-year-old female child was the first paediatric case, and the youngest paediatric case of COVID-19 as of 2 March 2020 was a 45 -day-old male baby. ${ }^{42}$ To date, the youngest reported paediatric case was a 36-hour-old baby in China. ${ }^{13}$

The median age of the paediatric patients with COVID-19 from Madrid, Spain was 1 year (range, 0-15 years). The largest dataset of 2143 paediatric patients from China reported the median age at diagnosis as 7 years. ${ }^{14}$ However, in the largest-todate American dataset of 2572 COVID-19 cases in children aged $<18$ years, the median age was reported as 11 years. ${ }^{10}$

Comparing the two large studies, ${ }^{10,14}$ we believe that there is variation in the median age between different regions of the world. However, paediatric data from other parts of the world to confirm this are still lacking. We deduce that children of all ages can be infected, including neonates, infants, and young children. 
TABLE I. Summary of clinical manifestations and outcomes of COVID-19 in the paediatric population*

\begin{tabular}{|c|c|c|c|c|c|c|c|c|}
\hline \multirow[t]{2}{*}{ Study } & \multirow{2}{*}{$\begin{array}{l}\text { No. of } \\
\text { patients } \\
\text { and sex }\end{array}$} & \multirow{2}{*}{$\begin{array}{l}\text { Age } \\
\text { (mean, } \\
\text { range) }\end{array}$} & \multirow{2}{*}{$\begin{array}{l}\text { Clinical } \\
\text { classification }\end{array}$} & \multirow{2}{*}{$\begin{array}{l}\text { Signs and } \\
\text { symptoms }\end{array}$} & \multicolumn{2}{|c|}{ Investigations } & \multirow[t]{2}{*}{ Management } & \multirow[t]{2}{*}{ Outcome } \\
\hline & & & & & Laboratory findings & $\begin{array}{l}\text { Radiological } \\
\text { findings }\end{array}$ & & \\
\hline $\begin{array}{l}\text { CDC } \\
\text { COVID-19 } \\
\text { Response } \\
\text { Team }^{10}\end{array}$ & $\begin{array}{c}2572 \\
M=1408 / \\
2490 \dagger \\
F=1082 / \\
2490\end{array}$ & $\begin{array}{c}11 \\
(0-17) y \ddagger\end{array}$ & NA & $\begin{array}{l}(\mathrm{n}=291) \dagger \\
\text { Fever: } 163(56 \%) \\
\text { Cough: } 158(54.3 \%) \\
\text { Dyspnoea: } 39(13.4 \%) \\
\text { Myalgia: } 66(22.7 \%) \\
\text { Nausea/vomiting: } \\
31(10.7 \%) \\
\text { Abdominal pain: } \\
17(5.8 \%) \\
\text { Diarrhoea: } 37(12.7 \%)\end{array}$ & NA & NA & NA & Died: 3 (0.12\%) \\
\hline $\begin{array}{l}\text { Tagarro et } \\
\text { al }^{11}\end{array}$ & $\begin{array}{c}41 \\
M=18 \\
F=23\end{array}$ & $\begin{array}{c}3 \\
(0-15) y \ddagger\end{array}$ & NA & NA & NA & NA & $\begin{array}{l}\text { High-flow } \\
\text { ventilation, } \\
\text { non-invasive and } \\
\text { invasive mechanical } \\
\text { ventilation }\end{array}$ & NA \\
\hline $\begin{array}{l}\text { Dong et } \\
\mathrm{al}^{14} \S\end{array}$ & $\begin{array}{c}2143 \\
M=1213 \\
F=930\end{array}$ & $7 y$ & $\begin{array}{l}\text { Mild: } \\
1091(50.9 \%) \\
\text { Moderate: } \\
831(38.8 \%) \\
\text { Severe: } \\
112(5.2 \%) \\
\text { Critical: } 13(0.6 \%)\end{array}$ & $\begin{array}{l}\text { Asymptomatic: } \\
94(4.4 \%)\end{array}$ & NA & NA & NA & Died: 1 (0.05\%) \\
\hline Qiu et $\mathrm{al}^{15}$ & $\begin{array}{c}36 \\
M=23 \\
F=13\end{array}$ & $\begin{array}{c}8.3 \\
(1-16) y\end{array}$ & $\begin{array}{l}\text { Mild: } 17(47.2 \%) \\
\text { Moderate: } \\
19(52.8 \%) \\
\text { Severe: } 0 \\
\text { Critical: } 0\end{array}$ & $\begin{array}{l}\text { Fever: } 13(36.1 \%) \\
\text { Cough: } 7(19.4 \%) \\
\text { Headache: } 3(8.3 \%) \\
\text { Sore throat: } 2(5.6 \%) \\
\text { Vomiting/diarrhoea: } \\
2(5.6 \%) \\
\text { Tachypnoea } \\
\text { and pharyngeal } \\
\text { congestion: } 1(2.8 \%)\end{array}$ & $\begin{array}{l}\text { Lymphopenia: } \\
11 \text { (30.6\%) } \\
\text { Leukopenia: } 7 \text { (19.4\%) } \\
\text { Elevated PCT: } \\
6 \text { (16.7\%) } \\
\text { Elevated CK-MB: } \\
11(30.6 \%) \\
\text { Elevated D-dimers / } \\
\text { ALT / AST: } 3(8.3 \%) / \\
2(5.6 \%) \text { / } 3(8.3 \%)\end{array}$ & $\begin{array}{l}\text { Chest CT } \\
\text { GGO: } 19 \text { (52.8\%) }\end{array}$ & $\begin{array}{l}\text { Interferon alpha, } \\
\text { lopinavir/ritonavir, } \\
\mathrm{O}_{2} \text { therapy }\end{array}$ & $\begin{array}{l}\text { Discharged: } \\
36(100 \%)\end{array}$ \\
\hline Xia et $a^{16}$ & $\begin{array}{l}20 \\
M=13 \\
F=7\end{array}$ & $\begin{array}{c}2.1 \\
(0-14) y \ddagger\end{array}$ & NA & $\begin{array}{l}\text { Cough: } 13(65 \%) \\
\text { Fever: } 12(60 \%) \\
\text { Diarrhoea: } 3(15 \%) \\
\text { Vomiting: } 2(10 \%) \\
\text { Moist rales: } 3(15 \%) \\
\text { Tachypnoea: } 2(10 \%) \\
\text { Fatigue: } 1(5 \%) \\
\text { Sore throat, cyanosis: } \\
1(5 \%)\end{array}$ & $\begin{array}{l}\text { Leukopenia: } 4 \text { (20\%) } \\
\text { Lymphopenia: } 7(35 \%) \\
\text { Elevated PCT / CRP / } \\
\text { ALT / CK-MB: } 16(80 \%) \text { / } \\
9(45 \%) \text { / } 5 \text { (25\%) / } \\
15(75 \%) \\
\text { Abnormal ECG: } 4(20 \%)\end{array}$ & $\begin{array}{l}\text { Chest CT } \\
\text { GGO: } 12(60 \%) \\
\text { Consolidation with } \\
\text { surrounding halo sign: } \\
10(50 \%) \\
\text { Fine mesh shadow: } \\
4(20 \%) \\
\text { Tiny nodules: } 3(15 \%)\end{array}$ & NA & $\begin{array}{l}\text { Discharged: } \\
18(90 \%) \\
\text { Admitted: } \\
2(10 \%)\end{array}$ \\
\hline Han et $\mathrm{al}^{18}$ & $\begin{array}{c}7 \\
M=4 \\
F=3\end{array}$ & $\begin{array}{c}1.3 \\
(0.2-13 y)\end{array}$ & $\begin{array}{l}\text { Mild: } 7(100 \%) \\
\text { Severe: } 0 \\
\text { Critical: } 0\end{array}$ & $\begin{array}{l}\text { Fever: } 5(71.4 \%) \\
\text { Cough: } 5(71.4 \%) \\
\text { Diarrhoea/vomiting: } \\
4(57.1 \%) \\
\text { Shortness of breath: } \\
3(42.9 \%) \\
\text { Pharyngalgia: } \\
1(14.3 \%)\end{array}$ & $\begin{array}{l}\text { Leukocytosis: } 2 \text { (28.6\%) } \\
\text { Elevated ESR: } \\
3(42.9 \%) \\
\text { Elevated CRP, LDH, } \\
\text { and D-dimer: } 2 \text { (28.6\%) } \\
\text { Elevated PCT / BNP: } \\
3 \text { (42.9\%) / } 5 \text { (71.4\%) } \\
\text { Elevated ALT / AST: } \\
1 \text { (14.3\%) / } 3 \text { (42.9\%) }\end{array}$ & $\begin{array}{l}\text { Chest CT and CXR } \\
\text { Normal: } 2(28.6 \%) \\
\text { GGO consolidation: } \\
5(71.4 \%)\end{array}$ & $\begin{array}{l}\mathrm{O}_{2} \text { therapy, } \\
\text { glucocorticoids }\end{array}$ & $\begin{array}{l}\text { Discharged: } \\
7(100 \%)\end{array}$ \\
\hline $\begin{array}{l}\text { Shen et } \\
\mathrm{al}^{19}\end{array}$ & $\begin{array}{c}9 \\
M=3 \\
F=6\end{array}$ & $\begin{array}{c}8 \\
(1-12) y \ddagger\end{array}$ & $\begin{array}{l}\text { Mild: } 5(55.6 \%) \\
\text { Moderate: } \\
2 \text { (22.2\%) } \\
\text { Severe: } 0 \\
\text { Critical: } 0\end{array}$ & $\begin{array}{l}\text { Asymptomatic: } \\
2(22.2 \%) \\
\text { Fever: } 3(33.3 \%) \\
\text { Diarrhoea: } 2(22.2 \%) \\
\text { Cough: } 1(11.1 \%)\end{array}$ & $\begin{array}{l}\text { Leukocytosis: } 1(11.1 \%) \\
\text { Elevated ESR: } \\
1(11.1 \%) \\
\text { Elevated CRP and AST: } \\
2(22.2 \%)\end{array}$ & $\begin{array}{l}\text { Chest CT } \\
\text { GGO: } 2(22.2 \%)\end{array}$ & $\begin{array}{l}\mathrm{O}_{2} \text { therapy, } \\
\text { lopinavir/ ritonavir, } \\
\text { azithromycin, IVIg, } \\
\text { glucocorticoids }\end{array}$ & $\begin{array}{l}\text { Discharged: } \\
6(66.7 \%) \\
\text { Admitted: } \\
3(33.3 \%)\end{array}$ \\
\hline Su et $\left.a\right|^{20}$ & $\begin{array}{c}9 \\
M=3 \\
F=6\end{array}$ & $\begin{array}{c}3.5 \\
(0.9-9) y\end{array}$ & NA & $\begin{array}{l}\text { Asymptomatic: } \\
6(66.7 \%) \\
\text { Fever: } 2(22.2 \%) \\
\text { Cough: } 1(11.1 \%)\end{array}$ & $\begin{array}{l}\text { Leukopenia: } 3 \text { (33.3\%) } \\
\text { Elevated CK-MB, ALT, } \\
\text { and AST: } 6(66.7 \%)\end{array}$ & $\begin{array}{l}\text { Chest CT and CXR } \\
\text { Normal: } 5(55.6 \%) \\
\text { Bronchitis: } 2(22.2 \%) \\
\text { Bronchopneumonia, } \\
\text { GGO } \pm \text { consolidation: } \\
1(11.1 \%)\end{array}$ & Interferon, ribavirin & $\begin{array}{l}\text { Discharged: } \\
9(100 \%) \\
\left(5 \text { re-admitted }{ }^{\star \star}\right)\end{array}$ \\
\hline
\end{tabular}

Abbreviations: ALT = alanine aminotransferase; $\mathrm{AST}=$ aspartate aminotransferase; BNP = brain natriuretic peptide; $\mathrm{CK}-\mathrm{MB}=$ creatine kinase myocardial band; COVID-19 = coronavirus disease 2019; CRP = C-reactive protein; CT = computed tomography; CXR =chest X-ray; DIC = disseminated intravascular coagulation; $\mathrm{ECG}=$ electrocardiogram; ESR = erythrocyte sedimentation rate; GGO = ground-glass opacities; ICU = intensive care unit; IL = interleukin; $\mathrm{IVIg}=$ intravenous immunoglobulin; $\mathrm{LDH}=$ lactate dehydrogenase; MODS = multiple organ dysfunction syndrome; $\mathrm{NA}=$ not available; O2 = oxygen;

$\mathrm{PCR}=$ polymerase chain reaction; $\mathrm{PCT}=$ procalcitonin

* Data are shown as No. (\%), unless otherwise specified

$\dagger$ Information about the remaining cases was not available

$\neq$ Values displayed as medians

$\S$ Both laboratory-confirmed and suspected cases were included. The percentage of confirmed cases was 34. $1 \%$

|| Based on "Recommendations for the diagnosis, prevention and control of the 2019 novel coronavirus infection in children (first interim edition)" 40

T Based on "Diagnosis and treatment scheme for new coronavirus infected pneumonia (fifth interim edition, revised)" 41

*** Positive stool PCR after discharge 
TABLE I. (cont'd)

\begin{tabular}{|c|c|c|c|c|c|c|c|c|}
\hline \multirow[t]{2}{*}{ Study } & \multirow{2}{*}{$\begin{array}{l}\text { No. of } \\
\text { patients } \\
\text { and sex }\end{array}$} & \multirow{2}{*}{$\begin{array}{l}\text { Age } \\
\text { (mean, } \\
\text { range) }\end{array}$} & \multirow{2}{*}{$\begin{array}{l}\text { Clinical } \\
\text { classification }\end{array}$} & \multirow{2}{*}{$\begin{array}{l}\text { Signs and } \\
\text { symptoms }\end{array}$} & \multicolumn{2}{|c|}{ Investigations } & \multirow[t]{2}{*}{ Management } & \multirow[t]{2}{*}{ Outcome } \\
\hline & & & & & Laboratory findings & $\begin{array}{l}\text { Radiological } \\
\text { findings }\end{array}$ & & \\
\hline $\begin{array}{l}\text { Zheng et } \\
\mathrm{al}^{21}\end{array}$ & $\begin{array}{c}25 \\
M=14 \\
F=11\end{array}$ & $\begin{array}{c}3 \\
(0.25-14) y\end{array}$ & $\begin{array}{l}\text { Upper respiratory } \\
\text { infection: } 8(32 \%) \\
\text { Mild pneumonia: } \\
15(60 \%) \\
\text { Critical: } 2(8 \%)\end{array}$ & $\begin{array}{l}\text { Fever: } 13(52 \%) \\
\text { Cough: } 11(44 \%) \\
\text { Nasal congestion and } \\
\text { dyspnoea: } 2(8 \%) \\
\text { Abdominal pain, } \\
\text { vomiting: } 2(8 \%) \\
\text { Diarrhoea: } 3(12 \%)\end{array}$ & Lymphopenia: 10 (40\%) & $\begin{array}{l}\text { Chest CT } \\
\text { Normal: } 8 \text { (32\%) } \\
\text { GGO } \pm \text { consolidation: } \\
16(64 \%) \\
\text { Unilateral: } 5(20 \%) \\
\text { Bilateral: } 11(44 \%)\end{array}$ & $\begin{array}{l}\text { Antivirals (arbidol, } \\
\text { oseltamivir, } \\
\text { lopinavir/litonavir, } \\
\text { interferon), } \\
\text { antibiotics, IVIg, } \\
\text { invasive ventilation, } \\
\text { renal replacement } \\
\text { therapy, } \\
\text { glucocorticoids }\end{array}$ & $\begin{array}{l}\text { Recovered: } \\
25(100 \%) \\
\text { Discharged: } \\
1(4 \%)\end{array}$ \\
\hline $\begin{array}{l}\text { Wang et } \\
\mathrm{al}^{22}\end{array}$ & $\begin{array}{c}31 \\
M=15 \\
F=16\end{array}$ & $\begin{array}{c}7.1 \\
(0.5-17) y\end{array}$ & $\begin{array}{l}\text { Asymptomatic: } \\
4(12.9 \%) \\
\text { Mild: } 13(41.9 \%) \\
\text { Ordinary: } \\
14(45.2 \%) \\
\text { Severe: } 0 \\
\text { Critical: } 0\end{array}$ & $\begin{array}{l}\text { Fever: } 20(64.5 \%) \\
\text { Cough: } 14(45.2 \%) \\
\text { Rales: } 4(12.9 \%) \\
\text { Pharyngeal } \\
\text { congestion: } 3(9.7 \%) \\
\text { Fatigue and } \\
\text { headache: } 3(9.7 \%) \\
\text { Diarrhoea / vomiting: } \\
3 \text { (9.7\%) / } 2 \text { (6.5\%) } \\
\text { Runny nose and sore } \\
\text { throat: } 2(6.5 \%)\end{array}$ & $\begin{array}{l}\text { Leukocytosis: } 3 \text { (9.7\%) } \\
\text { Leukopenia and } \\
\text { lymphopenia: } 2 \text { (6.5\%) } \\
\text { Lymphocytosis: } \\
4(12.9 \%) \\
\text { Thrombocytosis: } \\
2(6.5 \%) \\
\text { Elevated PCT, ESR and } \\
\text { CK-MB: } 4 \text { (12.9\%) } \\
\text { Elevated CRP: } 3(9.7 \%) \\
\text { Elevated LDH and } \\
\text { D-dimers: } 2(6.5 \%)\end{array}$ & $\begin{array}{l}\text { Chest CT }(n=30) \\
\text { Normal: } 16(53.3 \%) \\
\text { Patchy GGO and } \\
\text { nodules: } 9(30 \%) \\
\text { CXR }(n=1) \\
\text { Visible weight gain in } \\
\text { both lungs }\end{array}$ & $\begin{array}{l}\text { Interferon and } \\
\text { oseltamivir alone or } \\
\text { in combination with } \\
\text { ribavirin (IV), abidol } \\
\text { (oral), lopinavir/ } \\
\text { ritonavir (oral), } \\
\text { IVlg, antibiotics, } \\
\text { decoction (oral } \\
\text { or IV) }\end{array}$ & $\begin{array}{l}\text { Recovered and } \\
\text { discharged: } \\
24(77.4 \%) \| \\
\text { Admitted: } \\
7(22.6 \%)\end{array}$ \\
\hline $\begin{array}{l}\text { Zhou et } \\
\mathrm{al}^{23}\end{array}$ & $\begin{array}{c}9 \\
M=4 \\
F=5\end{array}$ & $\begin{array}{c}1.57 \\
(0.6-3) y\end{array}$ & NA & $\begin{array}{l}\text { Asymptomatic: } \\
5 \text { (55.6\%) } \\
\text { Fever: } 4(44.4 \%) \\
\text { Cough: } 2(22.2 \%) \\
\text { Runny nose: } \\
1(11.1 \%)\end{array}$ & $\begin{array}{l}\text { Leukocytosis: } 2 \text { (22.2\%) } \\
\text { Lymphocytosis: } \\
6(66.7 \%) \\
\text { Elevated AST: } 4(44.4 \%) \\
\text { Elevated LDH and CRP: } \\
3(33.3 \%)\end{array}$ & $\begin{array}{l}\text { Chest CT } \\
\text { Patchy lesions: } \\
7 \text { (77.8\%) } \\
\text { Nodular lesions, GGO } \\
\pm \text { consolidation: } \\
6 \text { (66.7\%) } \\
\text { Halo sign: } 3(33.3 \%) \\
\text { Pleural effusion: } \\
1(11.1 \%)\end{array}$ & $\begin{array}{l}\text { Inhalational } \\
\text { interferon alone or } \\
\text { in combination with } \\
\text { lopinavir/ritonavir } \\
\text { (oral) }\end{array}$ & $\begin{array}{l}\text { Negative nucleic } \\
\text { acid: } 4(44.4 \%) \\
\text { Lung lesions } \\
\text { absorbed: } \\
3(33.3 \%)\end{array}$ \\
\hline $\begin{array}{l}\text { Feng et } \\
\mathrm{al}^{24}\end{array}$ & $\begin{array}{c}15 \\
M=5 \\
F=10\end{array}$ & $\begin{array}{c}7.8 \\
(4-14) y\end{array}$ & $\begin{array}{l}\text { Light: } 3(20 \%) \\
\text { Ordinary: } \\
12 \text { (80\%) } \\
\text { Heavy: 0 } \\
\text { Critical: 0ๆ }\end{array}$ & $\begin{array}{l}\text { Asymptomatic: } \\
8(53.3 \%) \\
\text { Fever: } 5(33.3 \%) \\
\text { Nasal congestion and } \\
\text { cough: } 1(6.7 \%)\end{array}$ & Leukopenia: 8 (53.3\%) & $\begin{array}{l}\text { Chest CT } \\
\text { Normal: } 6(40 \%) \\
\text { Nodular opacities: } \\
7 \text { (46.7\%) } \\
\text { GGO: } 2(13.3 \%)\end{array}$ & NA & $\begin{array}{l}\text { Recovered: } \\
15(100 \%) \\
\text { Discharged: } \\
5(33.3 \%)\end{array}$ \\
\hline Wei et $\mathrm{a}^{25}$ & $\begin{array}{c}9 \\
M=2 \\
F=7\end{array}$ & $\begin{array}{c}6.6 \\
(1-11) \mathrm{mo}\end{array}$ & NA & $\begin{array}{l}\text { Asymptomatic: } \\
1(11.1 \%) \\
\text { Fever: } 4(44.4 \%) \\
\text { Cough: } 2(22.2 \%)\end{array}$ & NA & NA & NA & NA \\
\hline Ma et $\mathrm{al}^{26}$ & $\begin{array}{c}6 \\
M=2 \\
F=4\end{array}$ & $\begin{array}{c}4.65 \\
(0.9-9) y\end{array}$ & NA & $\begin{array}{l}\text { Asymptomatic: } \\
4(66.7 \%) \\
\text { Fever: } 2(33.3 \%)\end{array}$ & NA & NA & NA & $\begin{array}{l}\text { Discharged: } \\
6(100 \%)^{\star \star}\end{array}$ \\
\hline Liu et $\mathrm{al}^{27}$ & $\begin{array}{c}6 \\
M=2 \\
F=4\end{array}$ & $\begin{array}{c}3.5 \\
(1-7) y\end{array}$ & $\begin{array}{l}\text { Moderate: } \\
5(83.3 \%) \\
\text { Severe: } 1(16.7 \%)\end{array}$ & $\begin{array}{l}\text { Fever: } 6(100 \%) \\
\text { Cough: } 6 \text { (100\%) } \\
\text { Vomiting: } 4(66.7 \%) \\
\text { Swollen tonsils: } \\
6 \text { (100\%) } \\
\text { Pharyngeal } \\
\text { congestion: } 5(83.3 \%) \\
\text { Wheezing: } 2(33.3 \%) \\
\text { Rhinorrhoea: } \\
1(16.7 \%) \\
\text { Chills: } 1(16.7 \%)\end{array}$ & $\begin{array}{l}\text { Lymphopenia: } 6(100 \%) \\
\text { Leukopenia: } 4(66.7 \%) \\
\text { Neutropenia: } 3(50 \%) \\
\text { Elevated CRP / } \\
\text { D-dimer: } 6 \text { (100\%) / } \\
3 \text { (50\%) } \\
\text { Elevated LDH and ESR: } \\
2 \text { (33.3\%) } \\
\text { Elevated AST / ALT: } \\
4(66.7 \%) / 1(16.7 \%)\end{array}$ & $\begin{array}{l}\text { Chest CT } \\
\text { Normal: } 1 \text { (16.7\%) } \\
\text { Patchy shadows: } \\
3 \text { (50\%) } \\
\text { Patchy GGO: } \\
1(16.7 \%)\end{array}$ & $\begin{array}{l}\text { Oseltamivir, } \\
\text { ribavirin, } \\
\text { glucocorticoids, } \\
\text { IVlg, } \mathrm{O}_{2} \text { therapy in } \\
\text { ICU patients }\end{array}$ & $\begin{array}{l}\text { Recovered: } \\
6(100 \%)\end{array}$ \\
\hline Sun et $\mathrm{al}^{28}$ & $\begin{array}{c}8 \\
M=6 \\
F=2\end{array}$ & $\begin{array}{c}6.8 \\
(0.2-15) y\end{array}$ & Severe: 8 (100\%) & $\begin{array}{l}\text { Polypnoea: } 8(100 \%) \\
\text { Fever: } 6 \text { (75\%) } \\
\text { Cough: } 6 \text { (75\%) } \\
\text { Expectoration: } \\
4 \text { (50\%) } \\
\text { Nausea/vomiting: } \\
4 \text { (50\%) } \\
\text { Diarrhoea: } 3(37.5 \%) \\
\text { Fatigue/myalgia: } \\
1 \text { (12.5\%) } \\
\text { Headache: } 1(12.5 \%) \\
\text { Rales: } 5(62.5 \%) \\
\text { Crackles: } 3(37.5 \%)\end{array}$ & $\begin{array}{l}\text { Leukocytosis: } 2 \text { (25\%) } \\
\text { Leukopenia: } 1 \text { (12.5\%) } \\
\text { Elevated CRP/LDH/ } \\
\text { PCT: } 5 \text { (62.5\%) } \\
\text { Elevated ALT: } 4 \text { (50\%) } \\
\text { Elevated CK-MB/ } \\
\text { D-dimer: } 2 \text { (25\%) } \\
\text { Cytokine assay } \\
\text { Elevated IL-10 / IL-6 / } \\
\text { interferon gamma: } \\
5 \text { (62.5\%) / } 2 \text { (25\%) / } \\
2 \text { (25\%) } \\
\text { Lymphocyte subset } \\
\text { analysis } \\
\text { Low CD16+ / CD56+ / } \\
\text { Th/Ts: } 4 \text { (50\%) / } \\
4 \text { (50\%) / } 1 \text { (12.5\%) } \\
\text { High CD3+ / CD4+ / } \\
\text { CD8+: } 2 \text { (25\%) / } \\
4 \text { (50\%) / } 1 \text { (12.5\%) }\end{array}$ & $\begin{array}{l}\text { Chest CT } \\
\text { Bilateral / unilateral } \\
\text { pneumonia: } 6(75 \%) \text { / } \\
2(25 \%) \\
\text { Multiple patch-like } \\
\text { shadows: } 7(87.5 \%) \\
\text { GGO: } 6(75 \%) \\
\text { Pleural effusion: } \\
1(12.5 \%) \\
\text { CXR } \\
\text { White lung-like } \\
\text { change: } 1(12.5 \%)\end{array}$ & $\begin{array}{l}\text { Antivirals, } \mathrm{O}_{2} \\
\text { therapy, antibiotics, } \\
\text { glucocorticoids, } \\
\text { IVlg, traditional } \\
\text { Chinese medicine, } \\
\text { invasive mechanical } \\
\text { ventilation, } \\
\text { plasmapheresis, } \\
\text { haemopurification, } \\
\text { transfusion }\end{array}$ & $\begin{array}{l}\text { Recovered and } \\
\text { discharged: } \\
5(62.5 \%) \\
\text { Admitted: } \\
3(37.5 \%) \\
\text { Complications } \\
\text { MODS and septic } \\
\text { shock: } 2(25 \%) \\
\text { DIC: } 1(12.5 \%), \\
\text { Septic } \\
\text { intussusception, } \\
\text { toxic } \\
\text { encephalopathy, } \\
\text { status epilepticus, } \\
\text { hydronephrosis, } \\
\text { cardiac } \\
\text { insufficiency, } \\
\text { coagulopathy }\end{array}$ \\
\hline
\end{tabular}


TABLE I. (cont'd)

\begin{tabular}{|c|c|c|c|c|c|c|c|c|}
\hline \multirow[t]{2}{*}{ Study } & \multirow{2}{*}{$\begin{array}{l}\text { No. of } \\
\text { patients } \\
\text { and sex }\end{array}$} & \multirow{2}{*}{$\begin{array}{l}\text { Age } \\
\text { (mean, } \\
\text { range) }\end{array}$} & \multirow{2}{*}{$\begin{array}{l}\text { Clinical } \\
\text { classification }\end{array}$} & \multirow{2}{*}{$\begin{array}{l}\text { Signs and } \\
\text { symptoms }\end{array}$} & \multicolumn{2}{|c|}{ Investigations } & \multirow[t]{2}{*}{ Management } & \multirow[t]{2}{*}{ Outcome } \\
\hline & & & & & Laboratory findings & $\begin{array}{l}\text { Radiological } \\
\text { findings }\end{array}$ & & \\
\hline $\begin{array}{l}\text { Zhang et } \\
\mathrm{al}^{29}\end{array}$ & $\begin{array}{c}3 \\
M=3 \\
F=0\end{array}$ & $\begin{array}{c}7.6 \\
(6-9) y\end{array}$ & Mild: 3 (100\%) & $\begin{array}{l}\text { Fever: } 2(66.7 \%) \\
\text { Nasal obstruction: } \\
2(66.7 \%) \\
\text { Gastrointestinal } \\
\text { symptoms: } 2 \text { (66.7\%) } \\
\text { Cough, wheezing, } \\
\text { expectoration: } \\
1(33.3 \%)\end{array}$ & $\begin{array}{l}\text { Leukocytosis: } 1(33 \%) \\
\text { Elevated CRP: } 1(33 \%) \\
\text { Low fibrinogen: } 2(67 \%)\end{array}$ & $\begin{array}{l}\text { Chest CT } \\
\text { GGO: } 2(67 \%) \\
\text { Cord shadow in lower } \\
\text { lung: } 1(33 \%)\end{array}$ & $\begin{array}{l}\text { Interferon } \\
\text { atomisation, } \\
\text { vitamin C, } \\
\text { oral traditional } \\
\text { Chinese medicine, } \\
\text { ceftriaxone }\end{array}$ & $\begin{array}{l}\text { Recovered and } \\
\text { discharged: } \\
3(100 \%)^{\star \star}\end{array}$ \\
\hline Hu et $\left.a\right|^{30}$ & $\begin{array}{c}6 \\
M=3 \\
F=3\end{array}$ & $\begin{array}{c}9.6 \\
(5-15) y\end{array}$ & NA & $\begin{array}{l}\text { Asymptomatic: } \\
5(83.3 \%)\end{array}$ & $\begin{array}{l}\text { Elevated PCT and LDH: } \\
2(33 \%) \\
\text { Elevated D-dimers / } \\
\text { ALT: } 1(17 \%) / 2(33 \%)\end{array}$ & $\begin{array}{l}\text { Chest CT } \\
\text { Normal: } 4(67 \%) \\
\text { GGO } \pm \text { consolidation: } \\
2(33 \%)\end{array}$ & $\begin{array}{l}\text { Antiviral, antibiotic, } \\
\text { antifungal, IVlg, } \\
\text { interferon }\end{array}$ & $\begin{array}{l}\text { Discharged: } \\
3 \text { (50\%) } \\
\text { Admitted: } \\
3(50 \%)\end{array}$ \\
\hline Li et $\mathrm{al}^{33}$ & $\begin{array}{c}5 \\
M=4 \\
F=1\end{array}$ & $\begin{array}{c}3.4 \\
(0.8-6) y\end{array}$ & NA & $\begin{array}{l}\text { Asymptomatic: } \\
4(80 \%) \\
\text { Fever, sore throat, } \\
\text { cough, sputum: } \\
1(20 \%)\end{array}$ & $\begin{array}{l}\text { Leukocytosis: } 2 \text { (40\%) } \\
\text { Elevated CRP: } 1 \text { (20\%) }\end{array}$ & $\begin{array}{l}\text { Chest CT } \\
\text { Patchy GGO: } 3(60 \%) \\
\text { Normal: } 2(40 \%)\end{array}$ & $\begin{array}{l}\text { IVlg, antivirals, } \\
\text { antibiotics, } \\
\text { montelukast, } \\
\text { Lianhua Qingwen } \\
\text { granules }\end{array}$ & $\begin{array}{l}\text { Discharged: } \\
3(60 \%) \\
\text { Admitted: } \\
2(40 \%)\end{array}$ \\
\hline Lu et $\mathrm{al}^{39}$ & $\begin{array}{c}171 \\
M=104 \\
F=67\end{array}$ & $\begin{array}{c}6.7 \\
(0-15) \mathrm{y} \ddagger\end{array}$ & $\begin{array}{l}\text { Asymptomatic } \\
\text { infection: } \\
27(15.8 \%) \\
\text { Upper respiratory } \\
\text { tract infection: } \\
33(19.3 \%) \\
\text { Pneumonia: } \\
111(64.9 \%)\end{array}$ & $\begin{array}{l}\text { Cough: } 83(48.5 \%) \\
\text { Fever: } 71(41.5 \%) \\
\text { Pharyngeal erythema: } \\
79(46.2 \%) \\
\text { Diarrhoea: } 15(8.8 \%) \\
\text { Fatigue: } 13(7.6 \%) \\
\text { Vomiting: } 11(6.4 \%) \\
\text { Tachycardia: } \\
72(42.1 \%) \\
\text { Tachypnoea: } \\
49(28.7 \%)\end{array}$ & $\begin{array}{l}\text { Leukopenia: } 45 \text { (26.3\%) } \\
\text { Lymphopenia: } 6 \text { (3.5\%) } \\
\text { Elevated PCT / CRP / } \\
\text { D-dimer: 105/164 } \\
\text { (64\%) / } 33(19.3 \%) / \\
21 / 149(14.1 \%) \\
\text { Elevated ALT / AST: } \\
21(12 \%) / 25(15 \%)\end{array}$ & $\begin{array}{l}\text { Chest CT } \\
\text { GGO: } 56(32.7 \%) \\
\text { Local patchy } \\
\text { shadowing: } \\
32(18.7 \%) \\
\text { Bilateral patchy } \\
\text { shadowing: } 21(12 \%) \\
\text { Interstitial } \\
\text { abnormalities: } \\
2(1.2 \%)\end{array}$ & Invasive ventilation & $\begin{array}{l}\text { Discharged: } \\
149(87 \%) \\
\text { Admitted: } \\
21(12 \%) \\
\text { Died: } 1(0.6 \%)\end{array}$ \\
\hline
\end{tabular}

\section{Sex}

An observational cohort study of 36 patients identified that $64 \%$ of the patients infected with SARS-CoV-2 were male. ${ }^{15}$ This is consistent with findings from other small studies. ${ }^{16,17}$ However, a large study by Dong et $\mathrm{al}^{14}$ did not find any statistically significant trend in the sex of paediatric patients, with boys comprising 1213 cases (56.6\%). This is in line with another large study from the US describing 2490 paediatric cases: $57 \%$ of the patients were male. ${ }^{10}$

\section{Incubation period}

The incubation period of COVID-19 ranged from less than 1 day to as long as 16 days. ${ }^{19}$ The median incubation period in children reported by Han et $\mathrm{al}^{18}$ and Shen et $\mathrm{al}^{19}$ was 5 days and 7.5 days, respectively.

\section{Symptoms}

Infected children were either asymptomatic or reported symptoms like fever and dry cough as the most common symptoms. ${ }^{13,15,19-26}$ Data reported by the US Centers for Disease Control and Prevention resonate with these findings: fever and cough were reported in $56 \%$ and $54 \%$ of the paediatric patients, respectively, whereas in another study, $68 \%$ of the paediatric cases had no obvious symptoms. ${ }^{10}$ A case series comprising of six patients reported fever and cough in all of them and vomiting in four of them. ${ }^{27}$ These results were confirmed by Xia et al, ${ }^{16}$ who found cough almost equally frequently as fever (65\% and 60\%, respectively). However, polypnoea has been reported as the most common symptom in severely affected patients, followed by fever and cough. ${ }^{28}$ A high proportion of patients presented initially with gastrointestinal symptoms such as nausea, vomiting, abdominal pain, constipation, and diarrhoea. ${ }^{10,15,16,18,19,21,22,27-29}$ Other reported symptoms were fatigue, myalgia, headache, ${ }^{10,16,18,22,28}$ and upper respiratory tract symptoms such as sore throat, nasal discharge, tachypnoea, and expectoration. ${ }^{10,13,15-17,19,21-25,28,29}$

Presentation of COVID-19 in paediatric patients is much milder than that in adults. Therefore, children may be a hidden source of infection. ${ }^{22}$ Fever, cough, and shortness of breath were more commonly reported among adult patients (93\%) than paediatric patients (73\%). ${ }^{10}$ Another study showed a statistically significant difference between symptomatic presentation in children and adults $(\mathrm{P}<0.0001)$ : fever ( $36 \%$ and $86 \%$, respectively), cough (19\% and 62\%), pneumonia (53\% and 95\%), and severe disease type ( $0 \%$ and $23 \%) .{ }^{15}$ In contrast, gastrointestinal symptoms were more common in children. ${ }^{10,18}$ Another difference was observed between the median duration of fever in children (1 day; range, 0-3 days) and adults (4 days; range, 1-10 days). ${ }^{18}$ 


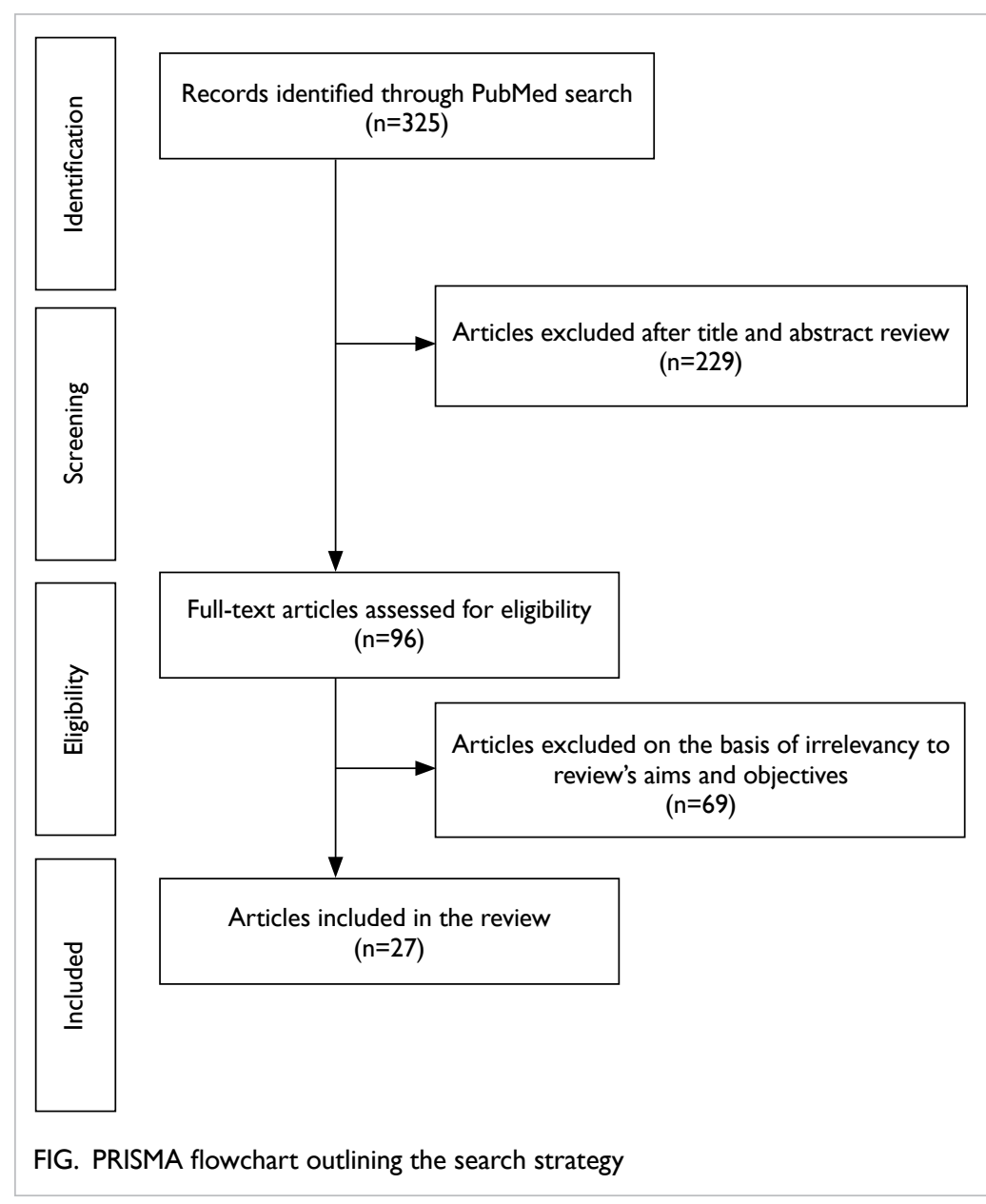

Dong et $\mathrm{al}^{14}$ reported that only $34.1 \%$ of cases were laboratory-confirmed, whereas the remainder had clinically suspected disease on the basis of their symptoms. However, this does not rule out their chance of other respiratory infections, so we cannot fully rely on clinical suspicion. Children with SARS-CoV, SARS-CoV-2, and H1N1 influenza presented with somewhat similar symptoms, with the most common being fever. However, cough and pharyngeal congestion are not as common in SARS-CoV-2 (7\% and 3\%, respectively) as in SARS-CoV (64\% and $14 \%)$ and H1N1 influenza ( $83 \%$ and $95 \%)$. These findings indicate that SARS-CoV-2 has little effect on the upper respiratory tract of children. . $^{15,24,25}$

There are many plausible explanations for why the disease's manifestations are milder in the paediatric population as compared with adults. First, it is very rare for children to have co-morbidities like diabetes, cardiovascular disease, and hypertension. Adults have a higher prevalence of C-reactive protein and longer duration of fever, suggesting a stronger immunological response compared with that in children. ${ }^{15,18}$ Further, children tend to remain at home and have fewer opportunities for exposure to pathogens or patients. Angiotensin-converting enzyme II is the receptor speculated to be affected by SARS-CoV-2. ${ }^{43,44}$ Children are less sensitive to COVID-19 because of their lower maturity and binding ability to a different distribution of angiotensin-converting enzyme II receptors. ${ }^{45}$ Furthermore, children experience respiratory infections in winter more often than adults do, which may result in higher levels of antibodies against other respiratory viruses, providing cross-protection against SARS-CoV-2. Additionally, children are mostly infected by the second or third generation of viral infections; hence, the viruses with which they are infected have weak virulence. Further, children's immune response is still under development and may show different responses to pathogens than that of adults. ${ }^{23,24} \mathrm{~A}$ different yet possible explanation is that like SARS and MERS-CoV infection in children, SARS-CoV-2 infection may follow a much milder and shorter course. ${ }^{21}$

\section{Radiological findings}

Among the studies that focused on radiographic findings in paediatric patients $(\mathrm{n}=224), 147(65.6 \%)$ patients showed positive findings suggestive of pneumonia. Most of the patients presented with abnormalities, such as multiple bilateral, peripheral ground-glass opacities (GGO), and consolidation. . $^{15,18-20,23,24,27,29,30}$ In comparison with adults, pulmonary inflammatory changes have been reported to be milder, ${ }^{15,23,24}$ and nodular changes (75\%), revealed as halo sign and air bronchogram sign on computed tomography (CT), are more common in paediatric patients. ${ }^{16,22,23}$ Therefore, these should be considered as typical signs in paediatric patients.

Chest CT images demonstrated bilateral lung involvement in about $70 \%$ of children aged $<3$ years, and unilateral lesions and normal lungs were reported more frequently in children aged $\geq 6$ years. ${ }^{21}$ Lesions were mainly distributed in the middle and outer bands of the lungs near the pleura. However, $53 \%$ had no obvious abnormalities. ${ }^{22}$ When another group of 24 asymptomatic carriers was reviewed, $29.2 \%$ had normal CT images ${ }^{30}$ Feng et al ${ }^{24}$ examined nine of the 15 confirmed paediatric patients, of whom $54 \%$ had no clear symptoms on admission. However, their CT results were typical of SARS-CoV-2 infection. Chest CT scans revealed improvement in children after 3 to 5 days of treatment. ${ }^{17,20,24,29}$ However, lesions were sometimes still visible on chest CT despite two consecutive negative nucleic acid tests. ${ }^{16}$

The severity of the lesions was limited in the early stage, but they increased in density as the disease advanced, involving multiple lobes bilaterally. ${ }^{16,22}$ Earlier in the course of disease, CT showed consolidation with surrounding halo sign, GGO, fine mesh shadow, and tiny nodules in $50 \%$, $60 \%, 20 \%$, and $15 \%$ of cases, respectively. ${ }^{16}$ In severely 
ill patients, bilateral multiple patch-like shadows, GGO, 'white lung' change accompanied by air bronchogram sign, and pleural thickening was the characteristic picture on CT. ${ }^{16,21,28}$ In neonates, chest $\mathrm{X}$-ray commonly showed GGO and blurred lung margins followed by bilateral pneumothorax and signs of neonatal respiratory distress syndrome. ${ }^{17}$

Computed tomography features could play an important role in screening suspected cases radiographically while awaiting confirmation by real-time reverse transcription-polymerase chain reaction $(\mathrm{PCR})$, the results of which could be used to decide the subsequent plan of action. Computed tomography can also be useful in cases that yield multiple false-negative real-time reverse transcription-PCR tests despite being clinically symptomatic.

\section{Laboratory findings}

Analysis of laboratory tests has revealed different laboratory parameters in children compared with adults. In children, the peripheral white blood cell count and absolute lymphocyte count are usually normal or slightly reduced. ${ }^{11,15,19,20,22,24,27,28,29,31}$ In contrast, laboratory analyses of adults have shown low leukocyte counts and significant reductions in absolute peripheral blood lymphocyte counts. One study revealed leukopenia as a common finding among adults (20\%), whereas leukocytosis was more frequent in children $(28.6 \% ; \mathrm{P}=0.014) .{ }^{18}$ One study found an elevated lymphocyte count on the initial routine blood test in $66 \%$ of paediatric subjects. ${ }^{23}$ Although the association is not clear, this altered immune response and lack of significant lymphopenia might help to cause the milder presentation in children. Some features differed significantly according to disease severity. Sun et $\mathrm{al}^{28}$ observed normal or mildly raised levels of leukocytes, neutrophils, and lymphocytes in severely ill patients, whereas low counts were observed in critically ill patients with serious complications. Abnormalities in the cytokine spectrum, characterised by increased plasma concentrations of inflammatory cytokines, were seen more frequently in critically ill than severe patients. $^{28}$

Inflammatory markers like $\mathrm{C}$-reactive protein and erythrocyte sedimentation rate were normal or transiently elevated. ${ }^{19,22,23,38}$ Raised levels of procalcitonin (PCT) were linked with severe disease in children. ${ }^{11,16,22}$ The erythrocyte sedimentation rate was raised significantly in adults as compared with children $(\mathrm{P}=0.047)$, whereas $\mathrm{PCT}$ was elevated in $42.9 \%$ of children but no adult patients $(\mathrm{P}=0.007) .{ }^{18}$ Elevated PCT in children may indicate bacterial co-infection, and timely administration of antibiotics might prove beneficial.

Another characteristic feature of COVID-19 is that it affects vital organs like the lungs, liver, and heart, indicated by increased levels of myocardial enzymes, aspartate aminotransferase, alanine aminotransferase, and D-dimer. Myocardial zymography revealed a higher frequency of elevated levels of isoenzyme in children than in adults. ${ }^{11,16,18,20,28}$ The level of creatine kinase, an indicator of myocardial injury, is significantly higher in severely ill patients. ${ }^{28}$ Moreover, brain natriuretic peptide has been found in a few paediatric patients. ${ }^{28}$ The presence of creatine kinase and brain natriuretic peptide indicates that SARS-CoV-2 has the potential to cause heart injury. Therefore, attention should be paid to those laboratory results.

In summary, the laboratory findings reported in children with SARS-CoV-2 are inconsistent with those observed in adult cases. Disease progression is characterised by amplified inflammatory response or cytokine storm. Monitoring of laboratory parameters is suggested to identify patients who might show improvement with anti-inflammatory treatments.

\section{Treatment}

The principles of early identification, early isolation, early diagnosis, and early treatment should be stressed. Our review did not identify any treatment protocols or trials specific to the paediatric population. Although most children with mild disease may not have indications for hospitalisation, supervision must be ensured to contain and prevent transmission. As no vaccine is currently available, management plans include bed rest and supportive treatments like maintenance of water electrolyte balance and homeostasis, administration of antipyretics, and administration of broad-spectrum antibiotics because of the probability of coinfection. ${ }^{10,16,19-22,27,29-34,38}$

Given by spray or nebulisation in the early phase of disease, interferons, alone or in combination with other antivirals, have been shown to improve symptoms. ${ }^{10,11,20,22,23,29,30,33,38}$ Oral lopinavir/ritonavir or ribavirin have been used; however, their efficacy and safety remain to be determined. . $^{10,11,19,22,23,27,29,30,33,34,38}$ Corticosteroids and intravenous immunoglobulin have been used in severe cases only. ${ }^{10,18,19,22,27,33,38}$ Use of steroids for treatment of SARS-CoV and MERS-CoV resulted in increased rates of secondary bacterial and fungal infections and longer duration of hospital stay. Thus, in addition to suppressing the inflammatory response, steroids also delay viral clearance ${ }^{46}$ Because of the lack of evidence regarding efficacy, the World Health Organization's interim guidance advised against the use of steroids for treatment of novel coronavirus, unless indicated. ${ }^{47}$ For such cases, it is recommended to use steroids only in the short term, and only as a part of a clinical trial, to efficiently weigh their harms and benefits. ${ }^{48}$ Patients with COVID-19 should be closely 
monitored for signs of clinical deterioration, such as rapidly progressive respiratory failure, central cyanosis, coma, convulsion, and sepsis. If respiratory distress develops despite the use of a nasal catheter or mask oxygenation, a heated humidified high-flow nasal cannula and non-invasive ventilation should be used to target $\mathrm{SpO} 2 \geq 94 \%$. $^{9,11,17-19,27,32,42}$ Mechanical ventilation with endotracheal intubation should be adopted when no improvement is seen. ${ }^{10,11,38}$ Increased levels of pro-inflammatory factors have been seen in children. ${ }^{11,16,19,20,22-24,27-29,31,38}$ Thus, targeted anti-inflammatory therapies that might help with early control of disease progression are warranted in the future. Monitoring patients conditions closely and the application of timely and effective therapeutic protocols through multidisciplinary approaches could serve as the cornerstones of COVID-19 treatment.

\section{Co-morbidities}

Compared with adults, children rarely had co-morbidities. ${ }^{15,18}$ Zheng et $\mathrm{al}^{21}$ reported two patients with congenital heart disease, one of whom also had malnutrition and metabolic diseases. Among 345 paediatric cases with information on underlying conditions, $23 \%$ had at least one underlying condition, with chronic lung disease being the most common, followed by cardiovascular and immunosuppressive diseases. ${ }^{10}$ Tagarro et $\mathrm{al}^{11}$ reported that $27 \%$ of patients had underlying disease. Furthermore, Xia et $\mathrm{al}^{16}$ reported that $35 \%$ patients had a history of underlying diseases, which may indicate that such patients are more susceptible to SARS-CoV-2.

\section{Outcomes}

Although paediatric patients are susceptible to COVID-19, the case fatality rate of severe paediatric patients is much lower than that of adults (49.0\%), ${ }^{15}$ which indicates that they have favourable outcomes compared with adults. ${ }^{21}$ Paediatric patients mostly recover in 1 to 3 weeks and are generally discharged after consecutive negative nucleic acid tests. ${ }^{15,16,18-22,24,27,29-31,33}$ Tagarro et $\mathrm{al}^{11}$ reported that $60 \%$ of paediatric patients were hospitalised, with only $10 \%$ admitted to paediatric intensive unit care. Sun et $\mathrm{al}^{28}$ also reported that most severely ill patients recovered and were discharged. Another two children who received paediatric intensive unit care recovered without any adverse outcomes reported. ${ }^{21}$

Disease duration is relative to the severity of the disease: the duration is over 10 days across all patients and over 20 days in critically ill patients. ${ }^{28}$ Qiu et al ${ }^{15}$ concluded that patients with the moderate clinical type spent more days in hospital compared with those with mild clinical type $(\mathrm{P}=0.017)$. The length of hospital stay has varied between different studies: the minimum and maximum averages reported have been $8.3^{27}$ and 15.3 days, ${ }^{19}$ respectively. Because this parameter is multifactorial and influenced by isolation policy and the availability of health facilities and laboratory tests in different hospitals, the length of hospital stay does not necessarily predict the prognosis, and detailed analysis is expected on its significance.

Overall, the prognosis in neonates is also good. Most of them have been discharged after consecutive negative nucleic acid test results. ${ }^{13} \mathrm{~A}$ few have been kept under observation despite stable condition and negative clinical and radiological findings because of positive COVID-19 pharyngeal swab nucleic acid test results..$^{13,16,17}$

\section{Complications}

Almost all studies have reported recovery without any complications. In critically ill patients, septic shock and multiple organ dysfunction syndrome were the most common complications, and intussusception, toxic encephalopathy, status epilepticus, disseminated intravascular coagulation (DIC), hydronephrosis, cardiac insufficiency, coagulopathy, hypoglobulinaemia, and gastroenteritis were also reported. ${ }^{28}$ One study reported two critical cases with abnormal renal function and coagulapathy. ${ }^{21}$

\section{Deaths}

In China, the deaths of a 14-year-old boy ${ }^{14}$ and a 10-month-old baby with intussusception who developed multi-organ failure 4 weeks post-admission were reported. ${ }^{39}$ Three paediatric deaths were reported in the US..$^{10}$ None had been reported in Italy or Spain as of 15 March and 8 April 2020, respectively., ${ }^{911}$

\section{Window of stool polymerase chain reaction detection}

The ability of SARS-CoV-2 to infect the gastrointestinal tract is supported by detection of its nucleic acids in stool samples from adults and children. ${ }^{15,20,26,29,32}$ In spite of negative nucleic acid results from throat swab specimens, children's stools were still nucleic acid-positive after 10 days of recovery. ${ }^{29}$ Other studies have reported re-admission of discharged children with positive stool specimens but negative respiratory specimens. Although their prognosis is better than that of adults; the period of PCR positivity is longer in children. ${ }^{15,20,26}$ Poorer hand hygiene practices causing faecal-oral transmission might be a reason for the delayed clearance of viral RNA in children's stools. Although positive results cannot confirm that live virus is present in the stool, this still increases the infection risk to the public, so follow-up of specimen collection should be considered. The isolation period for children should be reviewed because of the transmission risk. 


\section{Transmission patterns}

The virus is mainly transmitted through respiratory droplets or contact. ${ }^{28}$ Transmission among the paediatric population mostly occurs by close contact with family members, ${ }^{14,18-23,29}$ a history of exposure to the epidemic area, or both. ${ }^{15,21}$ According to Wang et $\mathrm{al}^{22} 90 \%$ of cases were clustered in families. Another study reported that $62.5 \%$ cases were associated with familial clustering..$^{28}$ Neonates and infants are more likely than adults to be infected via close contact with COVID-19-positive family members. ${ }^{16,25}$

\section{Potential of intrauterine vertical transmission}

To date, nine studies have reported on neonates born to COVID-19 positive mothers ( $\mathrm{n}=115)$; only seven of those neonates were SARS-CoV-2-positive. ${ }^{13,16,32}$ $\mathrm{Xia}$ et $\mathrm{al}_{,}{ }^{16} \mathrm{Yu}$ et $\mathrm{al},{ }^{13}$ and Zeng et $\mathrm{al}^{32}$ reported that $3 / 20(15 \%), 1 / 7(14 \%)$, and $3 / 33(9 \%)$ neonates were positive, respectively. Despite the placenta and cord blood being negative for SARS-CoV-2, one neonate was diagnosed as positive 36 hours after birth. ${ }^{13}$ Other studies have also found that the placenta, cord blood, and breast milk were negative for SARS-CoV-2. ${ }^{17,31,34,37}$ Because of the limited evidence regarding vertical transmission, we speculate that close contact could explain the positive results. We are still not confident about the probability of vertical maternal-fetal transmission, and further studies need to be performed on this subject. Neonates born to infected mothers should be separated immediately after birth and undergo an isolation period.

\section{Impact of COVID-19 on fetal outcomes}

Although the majority of the studies showed that neonates were negative for SARS-COV-2 and that

TABLE 2. Outcomes of neonates born to COVID-19-positive mothers*

\begin{tabular}{|c|c|c|c|c|c|c|c|c|c|}
\hline & Yu et al $^{13}$ & Xia et al $^{16}$ & Zhu et al ${ }^{17}$ & Chen et $a^{31}$ & Zeng et $\mathrm{al}^{32}$ & Li et $a^{34}$ & Zhang et $\mathrm{al}^{35}$ & Chen et $a^{36}$ & Fan et $a^{37}$ \\
\hline No. of neonates & 7 & 3 & 10 & 9 & 33 & $36 \S$ & 10 & 5 & 2 \\
\hline $\begin{array}{l}\text { Gestational age at } \\
\text { delivery (weeks) }\end{array}$ & $\begin{array}{c}39+1 \\
(37 \text { to } 41+2)\end{array}$ & NA & $31-39$ & $36-39+4$ & $\begin{array}{c}37+2(31+2 \text { to } \\
40+4)\end{array}$ & $\begin{array}{c}38 \pm 0.2 \text { to } \\
38 \pm 2.9\end{array}$ & $\begin{array}{l}38.7 \pm 1.4 \\
(35+5 \text { to } 41)\end{array}$ & $38+6$ to $40+4$ & 37,36 \\
\hline $\begin{array}{l}\text { Premature rupture } \\
\text { of membrane }\end{array}$ & 0 & NA & $3(30 \%)$ & 2 (22.2\%) & $3(9.1 \%)$ & $2(5.6 \%)$ & $3(30 \%)$ & 0 & 0 \\
\hline $\begin{array}{l}\text { Preterm labour/ } \\
\text { delivery }\end{array}$ & 0 & NA & $6(60 \%)$ & $4(44.4 \%)$ & $4(12.1 \%)$ & $6(16.7 \%)$ & $3(30 \%)$ & 0 & 0 \\
\hline Caesarean section & $7(100.0 \%)$ & NA & $7(70 \%)$ & $9(100.0 \%)$ & $26(78.8 \%)$ & $30(83.3 \%)$ & $10(100 \%)$ & $5(100 \%)$ & $2(100 \%)$ \\
\hline $\begin{array}{l}\text { Birth weight } \\
\text { (mean, range) [g] }\end{array}$ & $\begin{array}{c}3264.28 \\
(3000-3500)\end{array}$ & NA & $\begin{array}{c}2423 \\
(1520-3800)\end{array}$ & $\begin{array}{c}3011.11 \\
(1880-3820)\end{array}$ & $\begin{array}{c}2730 \\
(1580-3360)\end{array}$ & $\begin{array}{c}3066.7 \pm 560.2 \\
\text { to } 3198.7 \pm \\
522.6\end{array}$ & $3139 \pm 437$ & $\begin{array}{c}3691 \\
(3235-4050)\end{array}$ & 3400,2890 \\
\hline $\begin{array}{l}\text { Apgar score at } 1 \\
\text { minute }\end{array}$ & $8-9$ & NA & $\begin{array}{c}10(n=1) \\
9(n=5) \\
8(n=3) \\
7(n=1)\end{array}$ & $8-9$ & $3(n=1) \ddagger$ & $9.6 \pm 0.5$ & NA & $10(n=5)$ & $9(n=2)$ \\
\hline $\begin{array}{l}\text { Apgar score at } 5 \\
\text { minutes }\end{array}$ & $8-9$ & NA & $\begin{array}{c}10(n=1) \\
9(n=5) \\
8(n=3) \\
7(n=1\end{array}$ & $9-10$ & $4(n=1) \ddagger$ & $10.0 \pm 0.0$ & NA & $10(n=5)$ & $10(n=2)$ \\
\hline Fetal distress & 0 & NA & $6(60 \%)$ & $2(22.2 \%)$ & $4(12.1 \%)$ & $3(8.3 \%)$ & $1(10.0 \%)$ & 0 & 0 \\
\hline Positive PCR & $\begin{array}{c}1(16.7 \%) \\
\text { Not tested: } 4 \\
(57.14 \%)\end{array}$ & $3(100 \%)$ & $\begin{array}{c}0 \\
\text { Not tested: } 1 \\
(10 \%)\end{array}$ & 0 & $3(9.1 \%)$ & 0 & 0 & 0 & 0 \\
\hline $\begin{array}{l}\text { Neonatal } \\
\text { complications }\end{array}$ & 0 & NA & $\begin{array}{c}\text { MODS: } 1 \\
(10.0 \%) \text { DIC: } 2 \\
(20.0 \%)\end{array}$ & 0 & $\begin{array}{c}\text { Feeding } \\
\text { intolerance: } 3 \\
(9.1 \%)\end{array}$ & 0 & 0 & 0 & 0 \\
\hline Neonatal asphyxia & 0 & NA & 0 & 0 & $2(6.1 \%)$ & 0 & $1(10.0 \%)$ & 0 & 0 \\
\hline Stillbirth & 0 & 0 & 0 & 0 & 0 & 0 & 0 & 0 & 0 \\
\hline Neonatal death & 0 & 0 & $1(10.0 \%) \dagger$ & 0 & 0 & 0 & 0 & 0 & 0 \\
\hline
\end{tabular}

Abbreviations: COVID-19 = coronavirus disease 2019; DIC = disseminated intravascular coagulation; MODS = multiple organ dysfunction syndrome; NA = not available; $P C R=$ polymerase chain reaction

* Data are shown as No. (\%), unless otherwise specified

$\uparrow$ The neonate died secondary to refractory shock and gastric bleeding

‡ Apgar scores of the remaining neonates were not available

$\S$ In total, 16 confirmed and 18 suspected cases were included in the study 
most neonates had excellent outcomes, ${ }^{36}$ a few studies revealed that neonates born to COVID-19positive mothers could develop other complications that could lead to poor neonatal outcomes. The rate of premature birth among newborns born to mothers with confirmed COVID-19 pneumonia (23.5\%) was significantly higher than the 2020 and 2019 control rates (5.8\% and $5.0 \%$, respectively). Low birth weight was also more frequent in infants of infected mothers $(17.6 \%)$ than in the control group $(2.5 \%) .^{34}$ Some incidence of premature birth, fetal distress, premature rupture of membranes, small size for gestational age, and large size for gestational age was observed in neonates born to COVID-19-positive mothers. ${ }^{17,35}$ Two COVID-19-positive neonates developed DIC, of whom one died on the 9th day secondary to refractory shock, multiple organ dysfunction syndrome, and DIC. ${ }^{17}$ The neonatal outcomes are summarised in Table 2. ${ }^{13,16,17,31,32,34-37}$

\section{Conclusion}

Paediatric patients make up a small fraction of COVID-19 cases, and they have a better prognosis than adult patients have. The differences in the mechanisms behind COVID-19's clinical manifestations between children and adults need to be verified by large, well-designed studies. Children are likely to become a hidden source of infection, which may delay the diagnosis of COVID-19, leading to unfavourable outcomes and causing community transmission. The probability of intrauterine vertical transmission in neonates is low, and close contact is the only plausible explanation for the observed positive results in neonates.

\section{Author contributions}

$M$ Jahangir designed the study. All authors contributed to the acquisition and analysis of data, and wrote the manuscript. $M$ Jahangir had critical revision of the manuscript for important intellectual content. All authors had full access to the data, contributed to the study, approved the final version for publication, and take responsibility for its accuracy and integrity.

\section{Conflicts of interest}

The authors have disclosed no conflicts of interest.

\section{Funding/support}

This research received no specific grant from any funding agency in the public, commercial, or not-for-profit sectors.

\section{References}

1. World Health Organization. Coronavirus disease (COVID-19)-events as they happen. Rolling updates on coronavirus disease (COVID-19). Available from: https://www.who.int/emergencies/diseases/novelcoronavirus-2019/events-as-they-happen. Accessed 30
Apr 2020.

2. World Health Organization. Coronavirus disease 2019 (COVID-19) situation report-51. Available from: https://www.who.int/docs/default-source/coronaviruse/ situation-reports/20200311-sitrep-51-covid-19. pdf?sfvrsn=1ba62e57_10. Accessed 30 Apr 2020.

3. World Health Organization. Coronavirus disease 2019 (COVID-19) situation report-101. Available from: https://www.who.int/docs/default-source/coronaviruse/ situation-reports / 20200430 -sitrep-101-covid-19. pdf?sfvrsn=2ba4e093_2. Accessed 30 Apr 2020.

4. World Health Organization. WHO Director-General's opening remarks at the media briefing on COVID-193 March 2020. Available from: https://www.who.int/ dg/speeches/detail/who-director-general-s-openingremarks-at-the-media-briefing-on-covid-19---3march-2020. Accessed 30 Apr 2020.

5. Cascella M, Rajnik M, Cuomo A, Dulebohn SC, Di Napoli R. Features, evaluation and treatment coronavirus (COVID-19). StatPearls Publishing; 2020 Jan. Available from: https:// www.statpearls.com/as/infectious/52171/. Accessed 30 Apr 2020.

6. Mahase E. Coronavirus: COVID-19 has killed more people than SARS and MERS combined, despite lower case fatality rate. BMJ 2020;368:m641.

7. Worldometer. Age, sex, existing conditions of COVID-19 cases and deaths. Available from: https://www. worldometers.info/coronavirus/coronavirus-age-sexdemographics/. Accessed 30 Apr 2020.

8. Wu Z, McGoogan JM. Characteristics of and important lessons from the coronavirus disease 2019 (COVID19) outbreak in China: summary of a report of 72314 cases from the Chinese Center for Disease Control and Prevention. JAMA 2020;323:1239-42.

9. Livingston E, Bucher K. Coronavirus disease 2019 (COVID-19) in Italy. JAMA 2020;323:1335.

10. CDC COVID-19 Response Team. Coronavirus disease 2019 in children-United States, February 12-April 2, 2020. MMWR Morb Mortal Wkly Rep 2020;69:422-6.

11. Tagarro A, Epalza C, Santos M, et al. Screening and severity of coronavirus disease 2019 (COVID-19) in children in Madrid, Spain. JAMA Pediatr 2020;Apr 8. Epub ahead of print.

12. Health Advisory Platform by Ministry of National Health Services Regulations and Coordination, Government of Pakistan. COVID-19. Available from: http:/covid.gov.pk/ stats/pakistan. Accessed 5 May 2020.

13. Yu N, Li W, Kang Q, et al. Clinical features and obstetric and neonatal outcomes of pregnant patients with COVID-19 in Wuhan, China: a retrospective, single-centre, descriptive study. Lancet Infect Dis 2020;20:559-64.

14. Dong Y, Mo X, Hu Y, et al. Epidemiology of COVID-19 among children in China. Pediatrics 2020;145:e20200702.

15. Qiu H, Wu J, Hong L, Luo Y, Song Q, Chen D. Clinical and epidemiological features of 36 children with coronavirus disease 2019 (COVID-19) in Zhejiang, China: an observational cohort study. Lancet Infect Dis 2020;20:68996.

16. Xia W, Shao J, Guo Y, Peng X, Li Z, Hu D. Clinical and CT features in pediatric patients with COVID-19 infection: different points from adults. Pediatr Pulmonol 2020;55:1169-74.

17. Zhu H, Wang L, Fang C, et al. Clinical analysis of 10 
neonates born to mothers with 2019-nCoV pneumonia. Transl Pediatr 2020;9:51-60.

18. Han YN, Feng ZW, Sun LN, et al. A comparative-descriptive analysis of clinical characteristics in 2019-coronavirusinfected children and adults. J Med Virol 2020;Apr 6. Epub ahead of print.

19. Shen Q, Guo W, Guo T, et al. Novel coronavirus infection in children outside of Wuhan, China. Pediatr Pulmonol 2020;55:1424-9.

20. Su L, Ma X, Yu H, et al. The different clinical characteristics of corona virus disease cases between children and their families in China-the character of children with COVID-19. Emerg Microbes Infect 2020;9:707-13.

21. Zheng F, Liao C, Fan QH, et al. Clinical characteristics of children with coronavirus disease 2019 in Hubei, China. Curr Med Sci 2020;40:275-80.

22. Wang D, Ju XL, Xie F, et al. Clinical analysis of 31 cases of 2019 novel coronavirus infection in children from six provinces (autonomous region) of northern China [in Chinese]. Zhonghua Er Ke Za Zhi 2020;58:269-74.

23. Zhou Y, Yang GD, Feng K, et al. Clinical features and chest CT findings of coronavirus disease 2019 in infants and young children [in Chinese]. Zhongguo Dang Dai Er Ke Za Zhi 2020;22:215-20.

24. Feng K, Yun YX, Wang XF, et al. Analysis of CT features of 15 children with 2019 novel coronavirus infection [in Chinese]. Zhonghua Er Ke Za Zhi 2020;58:E007.

25. Wei M, Yuan J, Liu Y, Fu T, Yu X, Zhang ZJ. Novel coronavirus infection in hospitalized infants under 1 year of age in China. JAMA 2020;323:1313-4.

26. Ma X, Su L, Zhang Y, Zhang X, Gai Z, Zhang Z. Do children need a longer time to shed SARS-CoV-2 in stool than adults? J Microbiol Immunol Infect 2020;53:373-6.

27. Liu W, Zhang Q, Chen J, et al. Detection of COVID-19 in children in early January 2020 Wuhan, China. N Engl J Med 2020;382:1370-1.

28. Sun D, Li H, Lu X, et al. Clinical features of severe pediatric patients with coronavirus disease 2019 in Wuhan: a single center's observational study. World J Pediatr 2020;16:2519.

29. Zhang T, Cui X, Zhao X, et al. Detectable SARS-CoV-2 viral RNA in feces of three children during recovery period of COVID-19 pneumonia. J Med Virol 2020;92:909-14.

30. $\mathrm{Hu} \mathrm{Z}$, Song $\mathrm{C}$, Xu C, et al. Clinical characteristics of 24 asymptomatic infections with COVID-19 screened among close contacts in Nanjing, China. Sci China Life Sci 2020;63:706-11.

31. Chen H, Guo J, Wang C, et al. Clinical characteristics and intrauterine vertical transmission potential of COVID-19 infection in nine pregnant women: a retrospective review of medical records. Lancet 2020;395:809-15.

32. Zeng L, Xia S, Yuan W, et al. Neonatal early-onset infection with SARS-CoV-2 in 33 neonates born to mothers with COVID-19 in Wuhan, China. JAMA Pediatr 2020;174:7225.

33. Li W, Cui H, Li K, Fang Y, Li S. Chest computed tomography in children with COVID-19 respiratory infection. Pediatr Radiol 2020;50:796-9.

34. Li N, Han L, Peng M, et al. Maternal and neonatal outcomes of pregnant women with COVID-19 pneumonia: a casecontrol study. Clin Infect Dis 2020;Mar 30. Epub ahead of print.

35. Zhang L, Jiang Y, Wei M, et al. Analysis of the pregnancy outcomes in pregnant women with COVID-19 in Hubei Province [in Chinese]. Zhonghua Fu Chan Ke Za Zhi 2020;55:166-71.

36. Chen S, Liao E, Cao D, Gao Y, Sun G, Shao Y. Clinical analysis of pregnant women with 2019 novel coronavirus pneumonia. J Med Virol 2020;Mar 28. Epub ahead of print.

37. Fan C, Lei D, Fang C, et al. Perinatal transmission of COVID-19 associated SARS-CoV-2: Should we worry? Clin Infect Dis 2020;Mar 17. Epub ahead of print.

38. Moher D, Liberati A, Tetzlaff J, Altman DG, PRISMA Group. Preferred reporting items for systematic reviews and meta-analyses: The PRISMA Statement. PLoS Med 2009;6:e1000097.

39. Lu X, Zhang L, Du H, et al. SARS-CoV-2 infection in children. N Engl J Med 2020;382:1663-5.

40. Recommendations for the diagnosis, prevention and control of the 2019 novel coronavirus infection in children (first interim edition) [in Chinese]. Zhonghua Er Ke Za Zhi 2020;58(0):E004.

41. National Health Commission of the People's Republic of China. Diagnosis and treatment scheme for new coronavirus infected pneumonia (fifth interim edition, revised). Available from: http://www.gov.cn/zhengce/ zhengceku/2020-02/09/5476407/files/765d1e65b7d14430 81053c29ad37fb07.pdf. Accessed 30 Apr 2020.

42. Park JY, Han MS, Park KU, Kim JY, Choi EH. First pediatric case of coronavirus disease 2019 in Korea. J Korean Med Sci 2020;35:e124.

43. Zhou P, Yang XL, Wang XG, et al. A pneumonia outbreak associated with a new coronavirus of probable bat origin. Nature 2020;579:270-3.

44. Wrapp D, Wang N, Corbett KS, et al. Cryo-EM structure of the 2019-nCoV spike in the prefusion conformation. Science 2020;367:1260-3.

45. Fang F, Luo XP. Facing the pandemic of 2019 novel coronavirus infections: the pediatric perspectives [in Chinese]. Zhonghua Er Ke Za Zhi 2020;58:81-5.

46. Russell CD, Millar JE, Baillie JK. Clinical evidence does not support corticosteroid treatment for 2019-nCoV lung injury. Lancet 2020;395:473-5.

47. World Health Organization. Clinical management of severe acute respiratory infection (SARI) when COVID-19 disease is suspected: interim guidance. Available from: https://www.who.int/publications/i/item/clinicalmanagement-of-covid-19. Accessed 29 May 2020.

48. Shen K, Yang Y, Wang T, et al. Diagnosis, treatment, and prevention of 2019 novel coronavirus infection in children: experts' consensus statement. World J Pediatr 2020;16:22331. 